\title{
Response of the nose to exercise in healthy subjects and in patients with rhinitis and asthma
}

\author{
Joan Serra-Batlles, Josep M Montserrat, Joaquim Mullol, Eugeni Ballester, \\ Antoni Xaubet, César Picado
}

\begin{abstract}
Background - Although the nose and the bronchi are both involved in the process of regulating respiratory heat exchange, thermal changes may precipitate airway obstruction during exercise but rarely cause nasal obstruction in patients with rhinitis. The cause of the different response of the nose and bronchial tree has hardly been investigated. This study was performed to assess the response of the nose during exercise in the presence of rhinitis, asthma, and in normal controls. Methods - Ten healthy subjects (group 1), 15 patients with asthma and rhinitis (group 2), 10 with rhinitis only (group 3), and 11 with asthma only (group 4) were included in the study. Exercise was performed on a bicycle ergometer for six minutes, reaching a heart rate of $80 \%$ of predicted. Bronchial and nasal responses were measured by forced expiratory volume in one second $\left(F E V_{1}\right)$ and posterior rhinomanometry, respectively. A drop in the $\mathrm{FEV}_{1}$ of $20 \%$ or more was considered a positive exercise induced asthma challenge test.
\end{abstract}

Results - Heart rate and ventilation increased by a similar proportion in the four groups. The $\mathrm{FEV}_{1}$ significantly decreased in asthmatic patients (groups 2 and 4) but it did not change in healthy subjects (group 1) or in those with rhinitis (group 3). Thirteen asthmatic patients developed exercise induced asthma. Nasal patency increased with exercise by a similar proportion in all groups, and no differences were detected between those with rhinitis (groups 2 and 3 ) and those without (groups 1 and 4). Nasal patency had returned to basal values at 25 minutes after completion of exercise in the four groups. The nose of patients with exercise induced asthma, however, remained significantly more patent than in patients without exercise induced asthma between 10 and 30 minutes after exercise.

Conclusions - These results suggest that the nose responds differently from the bronchi during exercise induced airway obstruction: whereas the bronchial tree responds by becoming narrowed, the nose becomes more patent. These findings suggest that the mechanisms regulating the response of the nose to exercise are different from those involved in the response of the bronchial tree.

(Thorax 1994;49:128-132)
The lamina propria of the nasal mucosa contains capacitance vessels which are venous sinusoids that form the erectile elements of the nose. While the patency of the nasal cavities is regulated by the erectile tissue, the lumen of the bronchi varies, at least in part, according to relaxation or contraction of the smooth muscle contained in their walls.

In asthmatic patients exercise induces an initial brochodilation which is often followed by bronchospasm. The bronchospastic response usually occurs after stopping exercise. The severity of the asthmatic response increases with increasing work load and also with increasing duration of exercise. ${ }^{1}$ It is generally accepted that airway obstruction after exercise is related to the thermodynamic events that occur in the tracheobronchial tree during exercise. $^{23}$

Although the nose and the bronchi both participate in the conditioning of inhaled air, thermal changes during exercise may cause bronchoconstriction in asthmatic patients but rarely, if ever, cause nasal obstruction in patients with rhinitis. ${ }^{45}$ Interestingly, in asthmatic subjects with rhinitis, ${ }^{6}$ as in healthy subjects, ${ }^{7}$ nasal resistance decreases with exercise and remains low even though the patient may develop severe bronchospasm.

Although the possible mechanisms responsible for exercise induced asthma have been extensively investigated, the response of the nose to exercise has received much less attention. The effect of rhinitis on the nasal response to exercise in the presence or absence of bronchial asthma has only been evaluated in poorly defined groups of patients and for very short periods. In order to improve the understanding of the nasal response to exercise we studied 46 subjects divided into four groups: healthy subjects, patients with rhinitis alone, patients with asthma, and those with both asthma and rhinitis.

\section{Methods}

POPULATION

Forty six non-smoking subjects were included in the four groups of the study which comprised 10 healthy subjects (group 1), 15 patients with rhinitis and asthma (group 2), 10 patients with rhinitis only (group 3 ), and 11 with asthma only (group 4).

Patients with asthma had a typical history of paroxysms of dyspnoea, wheezing, and coughing which improved after the administration of a bronchodilator. An increase in the FEV, exceeding $15 \%$ predicted had been obtained at least once following $200 \mu \mathrm{g}$ salbutamol. None 
of the patients had evidence of any other lung disease. Seven were atopic, having at least one positive weal ( $>3 \mathrm{~mm}$ in diameter) on skin prick testing to a battery of common allergens (Leti SA, Barcelona, Spain). None were on regular treatment with sodium cromoglycate, oral or inhaled steroids, and none routinely used sustained release theophylline preparations. All patients were receiving inhaled bronchodilator treatment on an as needed basis. Any type of $\beta$ adrenergic treatment was stopped at least 12 hours before the study.

Patients with rhinitis reported a history of persistent nasal symptoms (perennial rhinitis) precipitated by infection, exposure to cold, or house dust. In five patients with a history of house dust allergy the skin prick test was positive for Dermatophagoides pteronyssinus. Six patients were receiving intermittent treatment with antihistamines and three were using inhaled budesonide. All treatment was discontinued at least one week before the study.

Fifteen asthmatic patients (seven with rhinitis and eight without rhinitis) had suffered asthma attacks precipitated by exercise.

Healthy subjects were recruited from the staff of our institution; none had any past or current history suggestive of rhinitis or asthma. One had a positive skin test for Dermatophagoides pteronyssinus.

None of the subjects had experienced an upper respiratory tract infection in the four weeks preceding the study.

Informed consent was obtained from each subject and the study protocol was approved by the reseach committee of our institution.

\section{EXERCISE TEST}

All exercise tests were performed on a bicycle ergometer (Erich Jaeger, Würzburg, Germany). Subjects exercised while inspiring room air via a mouthpiece connected to a two way valve (No 2700, Hans-Rudolph) and expired into a 2001 Douglas bag. The expired air was collected for one minute at baseline and during the last minute during exercise. The air collected in the bag was passed into a 3501 gasometer (Tissot, Warren E Collins, UK) and ventilation was then measured. The work load began at a low level and increased progressively during the first two minutes ( 50 watts every 30 seconds) until the subject reached a heart rate of $80 \%$ of predicted maximum. Exercise was maintained at this work load for four minutes. Heart rate was measured by a Hewlett-Packard monitor (Hewlett-Packard, California, USA). During the six minutes of bicycling the patients wore a noseclip because,

Mean ( $S D$ ) data of subjects in study

\begin{tabular}{lcccc}
\hline & Healthy subjects & Rhinitis and asthma & Rhinitis & Asthma \\
\hline Sex (M:F) & $8: 2$ & $9: 6$ & $9: 1$ & $7: 4$ \\
Age (years) & $26(7)$ & $25(9)$ & $24(7)$ & $26(10)$ \\
FEV $_{1}(\%)$ & $103(8 \cdot 5)$ & $81(21)^{*}$ & $105(16)$ & $89(18)^{*}$ \\
Nasal flow at $150 \mathrm{~Pa}\left(\mathrm{~cm}^{3} / \mathrm{s}\right)$ & $493(152)$ & $379(206)$ & $496(235)$ & $460(183)$ \\
\hline
\end{tabular}

${ }^{*} \mathrm{p}<0.005$ compared with healthy subjects and patients with rhinitis. in a previous study, Olson and Strohl $^{7}$ showed that the response of the nasal airway to exercise was independent of flow through the nose.

The FEV 1 was measured in triplicate before the exercise test, with the highest reading being taken as the baseline value (Sibel Datospir, Barcelona, Spain). Upon completion of exercise the best of two $\mathrm{FEV}_{1}$ measurements was recorded for analysis. Spirometric tests were performed at approximately $1,5,10,15$, 20, 25, 30, 35, 40, 50, and 60 minutes. Predicted values were taken from Roca and colleagues. ${ }^{8}$

Posterior rhinomanometry was used in the measurement of airway resistance. A soft plastic catheter with an internal diameter of $1 \mathrm{~cm}$ was passed posteriorly over the tongue to measure pharyngeal pressure. Transnasal pressure and nasal flow were measured using a rhinomanometer (Rhinotest uP, EGV Elektronic, Ludwisghafen, Germany). Transnasal pressure was determined by measuring the difference between the pressure in the pharynx with the pressure immediately in front of the nose using a pressure transducer (Validyne MP45 \pm 50 ). Nasal flow was measured with a close fitting face mask fitted with a Fleish no. 3 pneumotachograph. According to the recommended standard methods, the patency of the nasal cavity was expressed as the flow generated at a pressure of $150 \mathrm{~Pa}\left(\mathrm{~cm}^{3} / \mathrm{s}\right)$. An increase of flow represented a decrease in the airflow resistance of the nasal cavity. ${ }^{9}$ In a previous study we examined the variability of rhinomanometric measurements performing repetitive studies in nine subjects for three consecutive days and found an intrasubject coefficient of variation of $11.05 \% .^{10}$

Nasal airflow was measured before and after the completion of the exercise test. Rhinomanometric evaluations were made immediately before spirometric tests at approximately $1,3,5,7,10,13,15,20,25,30,35,40,50$, and 60 minutes.

\section{STATISTICAL ANALYSIS}

Changes in heart rate, ventilation, $\mathrm{FEV}_{1}$ and nasal flow among the four groups at the different periods of time were analysed by analysis of variance for repetitive measurements with two factors (time and the presence of rhinitis or asthma). The same test was used to analyse the effect of exercise induced asthma on the nasal response. Sheffe's contrast test was used to examine pairs of means when the analysis of variance was significant. Significance in all data analysis was considered to be $\mathrm{p}<0.05$.

\section{Results}

Details of the subjects are summarised in the table. Basal FEV 1 was significantly lower $(p<0.005)$ in the asthmatic patients (groups 2 and 4) than in the healthy subjects and those with rhinitis (groups 1 and 3 ). No significant differences were found in nasal patency among the four groups. 


\section{EXERCISE TEST}

There were no differences in baseline heart rate among the four groups. Heart rate increased during exercise in a similar manner in all subjects, suggesting that the work done was similar (data not shown). Basal ventilation was similar in all groups and increased in a similar proportion during exercise. There were no differences in ventilation during the last minute of exercise (data not shown).

\section{BRONCHIAL RESPONSE}

$\mathrm{FEV}_{1}$ did not change after exercise in the healthy subjects (group 1) or in the patients with rhinitis (group 3) but decreased significantly in those with asthma (fig 1). No differences were found in the airway responses between asthmatic patients, with and without rhinitis (data not shown). Thirteen $(50 \%)$ of the 26 asthmatic patients studied developed a drop in $\mathrm{FEV}_{1}$ of $20 \%$ or more from the baseline value and were considered positive for exercise induced asthma. Among the 15 patients with a positive clinical history for exercise induced asthma, 11 (five with asthma and rhinitis and six with asthma alone) showed a positive exercise challenge test. Only two patients with a negative clinical history developed bronchoconstriction after the exercise test.

\section{NASAL RESPONSE}

Although patients with rhinitis showed a lower nasal flow at $150 \mathrm{~Pa}$ pressure than the remaining three groups, the difference did not reach statistical significance (table). Nasal patency increased with exercise by a similar proportion in all groups and progressively returned to the normal range, returning almost to baseline values 25 minutes after the completion of exercise (fig 2). No differences in nasal responses

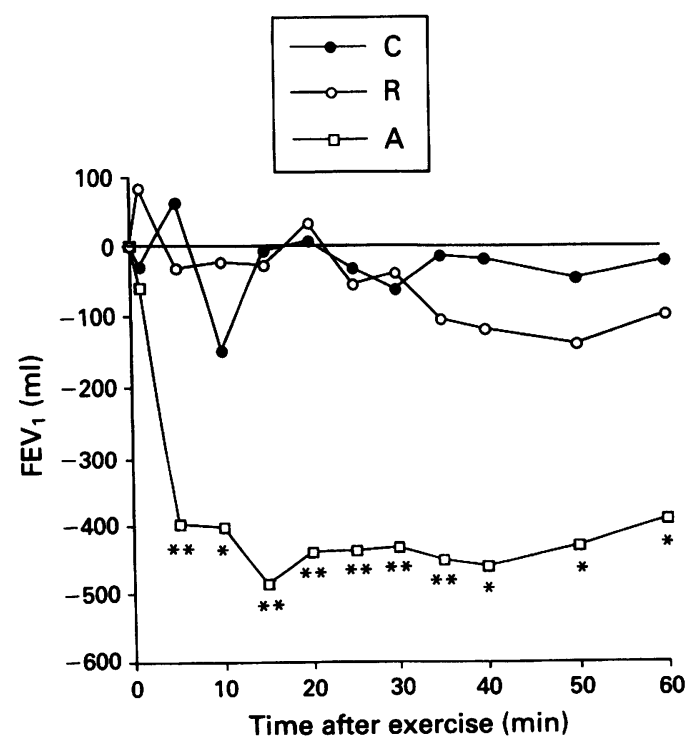

Figure 1 Changes in mean values of FEV, after exercise with respect to baseline values (represented by 0 line). No changes were found in non-asthmatic subjects (healthy subjects and those with rhinitis). A significant decrease was seen in patients with asthma. $C=$ control healthy subjects, $R=$ patients with rhinitis; $A=$ asthmatic patients (with and without rhinitis). ${ }^{*} p<0.05,{ }^{* *} p<0.01$.

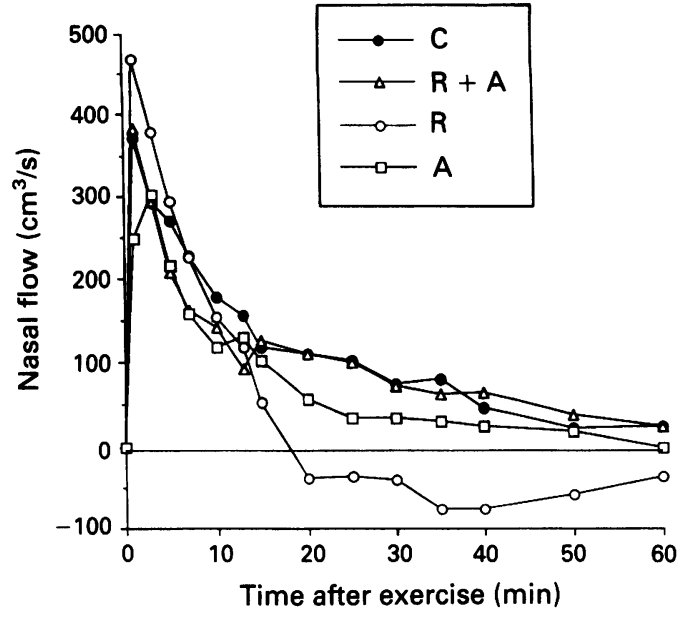

Figure 2 Changes in mean nasal flow at $150 \mathrm{~Pa}$ pressure after exercise with respect to baseline values (represented by line 0). No differences were found among healthy subjects, patients with rhinitis or asthma. $C=$ control healthy subjects; $R=$ patients with rhinitis; $A=$ patients with asthma.

were found when patients with and without rhinitis were grouped together, nor between asthmatic and non-asthmatic patients. However, the nasal response in asthmatic patients who developed exercise induced asthma differed from those who did not. In patients with exercise induced asthma nasal patency decreased at the same rate as non-responders during the first 10 minutes, but between 10 and 60 minutes the nose of patients with exercise induced asthma remained more patent and consequently the flow at $150 \mathrm{~Pa}$ was significantly higher in these patients than in the nonresponding asthmatics (fig 3 ). The intensity of exercise and baseline nasal airway resistance was similar in both groups of patients.

\section{Discussion}

Our study shows that the nose dilates during exercise and that this response is independent of the presence of rhinitis. We have also shown that, when exercise precipitates bronchocon-

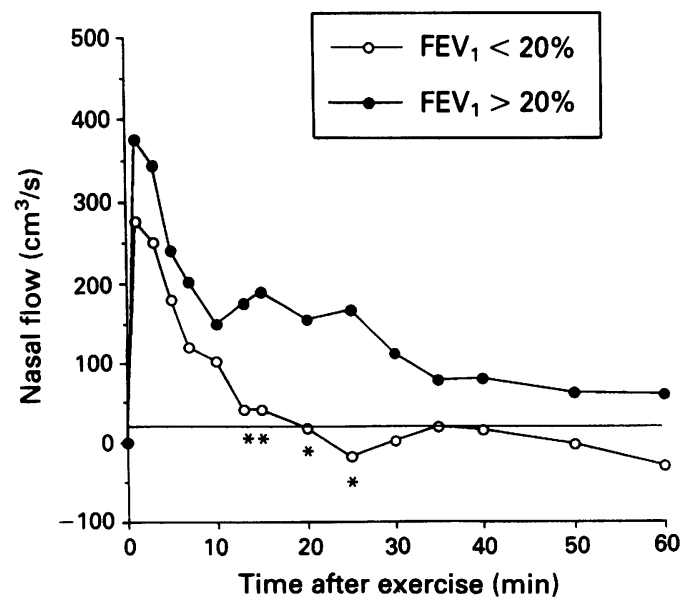

Figure 3 Changes in mean nasal flow at $150 \mathrm{~Pa}$ pressure after exercise with respect to baseline values (represented by 0 line) in patients with asthma. In patients with exercise induced asthma (a drop of the $F E V$, of $20 \%$ or more with respect to baseline values) the nasal flow remained significantly elevated compared with those without exercise induced asthma 15 to 25 minutes after completion of exercise. ${ }^{*} p<0.05$. 
striction in asthmatic subjects, the nasal response is completely dissociated from the bronchial response as the nose never develops obstruction. The nose remains significantly more dilated during bronchoconstriction, however, reinforcing the impression that it behaves inversely to the bronchi during exercise induced airway obstruction.

Why does the nose, which is morphologically and functionally closely linked to the upper and lower airways, behave in such a different manner? There is one study that might help to explain, at least in part, the peculiar response of the nose. Rubinstein et al ${ }^{11}$ investigated the response of the airways to exercise in asthmatic and healthy subjects using an acoustic reflection technique which allows the measurement of the diameter of the upper as well as the central airway. They found that, in healthy subjects, exercise did not change $\mathrm{FEV}_{1}$ but caused a significant increase of approximately $55 \%$ in the cross-sectional area of the intrathoracic trachea. No significant changes, however, were found in the bronchial tree. In asthmatic patients with exercise induced asthma, exercise resulted in a significant reduction in $\mathrm{FEV}_{1}$ and a concomitant decrease in the bronchial cross-sectional area: however, the extrathoracic and intrathoracic tracheal areas showed an unexpected and significant increase. These findings provide clear evidence that the bronchi are the main site of airway narrowing in exercise induced asthma and that tracheal dilation can occur at the same time. These observations fit well with our findings and suggest that the nasal response to exercise, both in healthy subjects and in patients with rhinitis and asthma, parallels that of the trachea. This increase in the upper airway diameter may explain the bronchodilator response usually detected immediately after exercise in both asthmatic and healthy subjects. ${ }^{1213}$ Taken together, these findings suggest that two different mechanisms regulate the patency of the upper and lower airways.

Although the role of vessels in the regulation of tracheal and main bronchi patency has received little attention, several studies have shown that the tracheobronchial mucosa has an extensive system of blood sinuses. ${ }^{14}$ Since the response of the trachea to exercise closely imitates the nasal response which is dependent on changes in the volume of capacitance vessels, it is reasonable to assume that the enlargement of the trachea during exercise might also be caused by a vasoconstictor phenomenon of the sinus network present in the tracheal mucosa.

Although at present there is no clear explanation for the dichotomous response of the different parts of the upper and lower airways, there is evidence in the literature to suggest that both parts show a different regulatory system. Sympathetic stimulation constricts the nasal blood vessels causing an increase in vascular resistance and nasal patency. The nasal response is abolished by stellate ganglion blockade with phentolamine. ${ }^{15}$ Laitinen et $a l^{16}$ have shown that alpha-adrenergic agonists reduce blood flow in the dog trachea, suggesting that both the nose and trachea are controlled by the same adrenergic system. In contrast with the upper airway, adrenergic nerve fibres seem to play a minor part in the regulation of lower airways in both man and animals. ${ }^{17}$

In our study we found that the nose remains significantly dilated in asthmatic patients who develop exercise induced asthma, suggesting the possible existence of a reflex from the lower airway to the nose. It is tempting to speculate that the open nose during exercise induced asthma observed in this study might represent a protective reflex mechanism to diminish airway resistance during peripheral airway obstruction. Syabbalo et $a l^{6}$ did not detect this response of the nose in asthmatic patients suffering exercise induced asthma, probably because they included in their study only four patients with exercise induced asthma in whom measurements of nasal flow were taken for 10 minutes after the completion of exercise. As differences in the nasal response between patients with exercise induced asthma and asthmatics without exercise induced asthma are detected after 10 minutes, this difference in methodology between the two studies probably accounts for the different results.

A rebound phenomenon characterised by an increase in nasal resistance starting approximately 20 minutes after exercise has been described in healthy subjects and in patients with either asthma or rhinitis. ${ }^{6}$ The mechanisms responsible for the rebound response are unknown. We did not detect any late increase in nasal airway resistance in our study. The rebound mainly occurs with mild degrees of exercise and is not usually detected when the work load is increased. ${ }^{6}$ Since our subjects were submitted to an intense level of exercise, this probably accounts for the lack of any late increase in nasal obstruction in our study.

In summary, we have shown that the nose, unlike the bronchi, never develops obstruction in patients suffering from rhinitis and asthma when challenged with exercise. We also found that the nose remains dilated during exercise induced bronchoconstriction in asthmatic patients. This finding suggests the presence of a reflex from the lower airway to the nose which increases nasal patency in these patients. Since a previous study has also shown a dichotomous response between the trachea and central/peripheral bronchi in exercise induced asthma, these results suggest that the mechanisms regulating the response of the upper airway (nose and trachea) to exercise differ from those controlling the central and peripheral airways.

1 Anderson SD. Exercise-induced asthma. The state of the art. Chest 1985;87S:191-5.

2 Anderson SD. Is there a unifying hypothesis for exerciseinduce asthma? F Allergy Clin Immunol 1984;73:660-5.

3 McFadden ER Jr. Hypothesis: exercise-induced asthma as a vascular phenomenon. Lancet 1990;335:880-3.

4 Gilbert IA, Fouke JM, McFadden ER Jr. Heat and water flux in the intrathoracic airways and exercise-induced flux in the intrathoracic airways and ex
asthma. $\mathcal{F}$ Appl Physiol 1987;63:1681-91.

5 Gilbert IA, Fouke JM, McFadden ER Jr. Intra-airway thermodynamics during exercise and hyperventilation in asthmatics. F Appl Physiol 1988;64:2167-74.

6 Syabbalo NC, Bundgaard A, Widdicombe JG. Effects of exercise on nasal airflow resistance in healthy subjects and in patients with asthma and rhinitis. Bull Eur Physiopathol Respir 1985;21:507-13. 
7 Olson LG, Strohl KP. The response of the nasal airway to exercise. Am Rev Respir Dis 1987;135:356-9.

8 Roca J, Sanchis J, Agusti-Vidal A, Segarra F, Navadas D, Rodriguez-Roisin R, et al. Spirometric reference values from a Mediterranean population. Bull Eur Physiopathol Respir 1986;22:217-24.

9 Schumacher MJ, Rhinomanometry. $\mathcal{f}$ Allergy Clin Immunol 1989;83:711-8.

10 Serra-Batlles J, Montserrat JM, Martos JA, Picado C. Rinomanometria. An Otorrinolaringol Ibero $\mathrm{Am}$ 1990;17:159-64.

11 Rubinstein I, Zamel N, Rebuck AS, Hoffstein V, D'Urzo $\mathrm{AD}$, Slutsky AS. Dichotomous airway response to exercise in asthmatic patients. Am Rev Respir Dis 1988;138:164-8.

12 Gelp AF, Tashkin DP, Epstein JD, Gong H, Zamel N.
Exercise-induced bronchoconstriction in asthma. Chest 1985;87:196-201.

13 Haas S, Pasierski S, Levine N, Bishop M, Axen K, Pineda $\mathrm{H}$, et al. Effect of aerobic training on forced expiratory airflow in exercising asthmatic humans. If Appl Physiol 1987;63:1230-5.

14 Widdicombe JG. Comparison between the vascular beds of upper and lower airways. Eur Respir $\mathcal{f}$ 1990;3(Suppl 12):564-75.

15 Richerson HB, Slebohm PM. Nasal airway response to exercise. $\mathcal{f}$ Allergy 1968;41:269-84.

16 Laitinen LA, Laitinen A, Widdicombe JG. Effects of inflammatory and other mediators on airway vascular beds. Am Rev Respir Dis 1987;135:67S-70S.

17 Doidge JM, Satchell DG. Adrenergic and non-adrenergic inhibitory nerves in mammalian airways. $f$ Auton Nerv Syst 1982;5:65-72. 